\title{
Quantum breathers in a nonlinear Klein Gordon lattice
}

\author{
Laurent Proville* \\ Service de Recherches de Métallurgie Physique, \\ CEA-Saclay/DEN/DMN 91191-Gif-sur-Yvette Cedex, France
}

(Dated: June 25, 2021)

\begin{abstract}
The quantum modes of a nonlinear Klein Gordon lattice have been computed numerically [L. Proville, Phys. Rev. B 71, 104306 (2005)]. The on-site nonlinearity has been found to lead to a phonon pairing and consequently some phonon bound states. In the present paper, the time dependent Wannier transform of these states is shown to exhibit a breather-like behavior, i.e., it is spatially localized and time-periodic. The typical time the lattice may sustain such breather states is studied as a function of the trapped energy and the intersite lattice coupling.

PACS numbers: 63.20.Ry, 03.65.Ge, 11.10.Lm, 63.20.Dj
\end{abstract}




\section{NONLINEAR LATTICE MODES}

The discrete breather solutions are currently a matter of intensive research (see Refs. $\left.{ }^{1.2 .3 .4 .5 .6 .7 .8 .9 .10 .11 .12 .13 .14}\right)$. The distinctive property of those lattice modes is to gather the spatial localization and the time periodicity so they lead to a energy trapping and thus a delay in the equipartition ${ }^{5}$. As a general consequence of anharmonicity, the emergence of breathers may be recognized as a paradigm of physics since it occurs at different scales in various contexts, e.g., in macroscopic networks as a chain of coupled pendulums, in microscopic Josephson arrays ${ }^{15}$ as well as in molecules ${ }^{16}$, polymers ${ }^{17}$ and crystals as the $\mathrm{PtCl}$ ethylene diamine chlorate $\frac{18}{}$.

The nonlinear excitations in materials have been studied for several decades. In the late fifties $\frac{19}{}$, the possible existence of a two-phonon bound state was pointed out in the infrared (IR) spectroscopy of $\mathrm{H}_{2}$ solid. The vibrational and rotational nonlinear excitations in the $\mathrm{H}_{2}$ crystal have been thoroughly investigated both experimentally ${ }^{20}$ and theoretically 21 . About the same period ${ }^{22}$, the spectrum anomalies of the crystalline acetanilide (ACN) was revealed and later interpreted with different theories (see Refs..$^{23.24 .25}$ and for a historical survey see $\left.\operatorname{Ref}^{26}\right)$. Early in the sixties ${ }^{27}$, in the $\mathrm{HCl}$ solid, the anharmonicity of the first overtone of hydrogen vibration has been measured by IR adsorption. It has been interpreted as a two phonon bound state, namely a biphonon ${ }^{28}$, in regard of the earlier theoretical work of V.M. Agranovich ${ }^{29.30}$. The triphonon has also been identified in the spectrum of $\mathrm{HCl}^{28}$. In seventies, similar phonon bound states have been recognized in several molecular crystals such as $\mathrm{CO}_{2}, \mathrm{~N}_{2} \mathrm{O}$ and $\mathrm{OCS}^{31}$, as well as in water ice ${ }^{32}$ by measuring the anharmonic IR absorptions. F. Bogani achieved some convincing simulations of these anharmonic spectra ${ }^{33}$ by using the technics of renormalized perturbation theory. For the last decade, the nonlinearity has emerged in several other materials:

- The inelastic neutron scattering (INS) has revealed the phonon bound states in the metal hydrides as $\mathrm{PdH}^{34}$ or $\mathrm{TiH}$ and $\mathrm{ZrH}^{35}$.

- The INS has also permitted to infer proton dynamics in the molecular crystals as polyglycine ${ }^{36}$ and 4-methylpyridine ${ }^{37}$. In the latter, the bound states of the methyl group rotational modes proved to last several days (see Refs. ${ }^{37.38}$ and Ref. ${ }^{26}$ for a survey of the theory). 
- The stretch overtone of carbon monoxide adsorbed on $\mathrm{Ru}(100)$ has been found to exhibit a strong anharmonicity at low surface coverage ${ }^{40.41}$. Several theoretical approaches $\stackrel{40,41.42}{\underline{n}}$ have been attempt to analyze the IR spectroscopy on $\mathrm{Ru}(100)$ : $\mathrm{CO}$.

The previous list is probably not complete but it is sufficient to emphasize that the nonlinear excitations have been worked out in many different materials, whether it is a molecular crystal ${ }^{33}$, a hydrogen-bonded crystal ${ }^{28}$ or a metal hydride ${ }^{34}$. Furthermore the nonlinearity may occur in one $\frac{37}{3}$, two $\underline{\underline{40}}$ and three-dimensiona ${ }^{33}$ systems. In most of the above cited examples, the phonon dispersion may be evaluated as smaller than $10 \%$ of the fundamental optical excitation and the anharmonicity proves to reach less than $5 \%$. The latter estimation holds for the first overtone whereas for higher orders the strength of the anharmonicity may increase as it is the case in $\mathrm{HCl}$ solid ${ }^{28}$ or stabilize as in $\mathrm{PdH}^{34}$. The phonon bound states are the siblings of breathers as they all stem from anharmonicity (see Refs. $\frac{14.26}{}$ and Refs. therein). The quantum breather may be viewed as a Wannier transform, applied to the phonon bound states that participate to a same energy band. Presently, the purpose of our work is to study this idea within the nonlinear KG model.

The accurate computation of nonlinear modes, whether it is in a classical lattice or in a quantum one requires the use of numerics. Recently $\stackrel{43}{\underline{4}}$, we proposed a numerical method that permits to compute the nonlinear quantum modes in a Klein Gordon lattice (KG) for different type of nonlinearity. In the present paper, we use those developments to study the Wannier transform $\frac{14}{\underline{4}}$ of the lattice eigen-modes that exhibits a quasi-particle spectrum, i.e., a narrow energy band. The time dependant Wannier transform of these states is found to exhibit a breather-like behavior, i.e., it is spatially localized and time-periodic. The life time of such breather states is studied as a function of their energy. We found that the higher the energy spike is, the longer it remains localized. That study has been carried out for different model parameters, including the case where the phonon dispersion is larger than the anharmonicity.

After a brief introduction of the nonlinear KG lattice model, our computing method is spelled out in Sec. III. In Sec. III and Sec. IV] we present and discuss our results on phonon bound states and breathers, respectively. Some perspectives are given in Sec. $\nabla$ 
FIG. 1: The plot of energy spectrum of a $1 \mathrm{D}$ chain, composed of $N=13$ atoms for $A_{4}=0.2$, versus the dimensionless coupling $C$. The eigenvalues are plotted as empty circles excepted the phonon bound states energies, plotted as full black circles. The tags indicate the order of phonon bound states.

\section{LATTICE MODEL AND NUMERICAL METHOD}

The energy of a lattice made of identical particles is expressed as a Hamiltonian operator:

$$
H=\sum_{l}\left[\frac{p_{l}^{2}}{2 m}+V\left(x_{l}\right)+\sum_{j=<l>} W\left(x_{l}-x_{j}\right)\right] .
$$

where $x_{l}$ and $p_{l}$ are displacement and momentum of the particle at site $l$, in a one-dimensional lattice. Such a lattice may prove relevant to model the quasi-one-dimensional networks of quantum particles in $\mathrm{ZrH}$ or in $\mathrm{PtCl}$. The quantum particle of mass $m$ evolves in a on-site potential $V$, being coupled to its nearest neighbors, $j=<l>$ by the interaction $W$. The on-site potential $V$ is developed to the fourth order whereas $W$ is modelled by a quadratic term:

$$
\begin{aligned}
& V\left(x_{l}\right)=a_{2} x_{l}^{2}+a_{3} x_{l}^{3}+a_{4} x_{l}^{4} \\
& W\left(x_{l}-x_{j}\right)=-c\left(x_{l}-x_{j}\right)^{2} .
\end{aligned}
$$

Higher order terms could have been added with no difficulty for our theory. It is possible to fixe the coefficients of $V$ within a first principle calculation as done for $\mathrm{PdH}^{44}$ and confirmed by the analyze of the INS spectrum ${ }^{34}$. For simplicity, we choose to fixe $a_{3}=0$. Introducing the dimensionless operators $P_{l}=p_{l} / \sqrt{m \hbar \Omega}, X_{l}=x_{l} \sqrt{m \Omega / \hbar}$ and the frequency $\Omega=\sqrt{2\left(a_{2}-2 . c\right) / m}$, the Hamiltonian is rewritten as follows:

$$
H=\hbar \Omega \sum_{l} \frac{P_{l}^{2}}{2}+\frac{X_{l}^{2}}{2}+A_{4} X_{l}^{4}+\frac{C}{2} X_{l} \sum_{j=<l>} X_{j}
$$

where the dimensionless coefficients are $A_{4}=a_{4} \frac{\hbar}{m^{2} \Omega^{3}}$ and $C=\frac{4 c}{m \Omega^{2}}$. The first step of our method is concerned with the exact diagonalization of the Hamiltonian where no interaction couples displacements. The procedure has been detailed in Ref. ${ }^{43}$. Arranging the on-site eigenvalues in increasing order, the $\alpha$ th eigenstate is denoted $\phi_{\alpha, i}$ and its eigenvalue is $\gamma(\alpha)$. In case of a negligible inter-site coupling, the $H$ eigenstates can be written as some Bloch 
FIG. 2: Energy spectrum of a 1D chain which model parameters are $A_{4}=0.2$ and $C=0.05$. The chain is composed of $N=13$ sites. Four energy regions have been reported: (a) phonons, (b) biphonons, (c) triphonons and (d) quadriphonons. The eigen-energies are plotted as empty symbols and the phonon bound states energies have been signalized by full symbols.

waves as follows:

$$
B_{\left[\Pi_{i} \alpha_{i}\right]}(q)=\frac{1}{\sqrt{A_{\left[\Pi_{i} \alpha_{i}\right]}}} \sum_{j} e^{-i q \cdot j} \Pi_{i} \phi_{\alpha_{i}, i+j}
$$

where $A_{\left[\Pi_{i} \alpha_{i}\right]}$ ensures the normalization. The label $\left[\Pi_{i} \alpha_{i}\right]$ identifies a single on-site state product $\Pi_{i} \phi_{\alpha_{i}, i}$ among the different products that may be derived from the present one by translation. The set of states $\left\{B_{\left[\Pi_{i} \alpha_{i}\right]}(q)\right\}_{q, N_{c u t}}$, including the uniform state $\Pi_{i} \phi_{0, i}$ at $q=0$, form a truncated basis where $N_{c u t}$ fixes the upper boundary on the on-site excitations: $\sum_{i} \alpha_{i} \leq N_{c u t}$. In case of a non-zero coupling, the states Eq.(4) may be thought as some Hartree approximation of the true eigenstates. The perturbation theory might be applied to the intersite coupling so as to estimate the eigenspectrum. However, we have chosen to carry out a computation as accurate as possible. Thus the Bloch wave basis is used to expand the Hamiltonian in. As the waves with different $q$, are not hybridized by $H$, the Hamiltonian can be expanded separately for each $q$. It can be achieved analytically whereas the diagonalization of the resulting matrix has been realized numerically with a standard method, from a numerical library ${ }^{45}$. The accuracy of our calculations has been tested both in a anharmonic ${ }^{43}$ and harmonic ${ }^{46}$ chain. For these two comparisons, a very good agreement has been found in the two-phonon energy region and lower.

We denote by $\psi_{\lambda}(q)$ and $E_{\lambda}(q)$ the $H$ eigenstates and the corresponding eigen-energies, respectively. The subscript $\lambda$ fixes the correspondence between a eigenstate and its eigenenergy. Our numerical technics allows us to compute the scalar product $V_{\lambda,\left[\Pi_{i} \alpha_{i}\right]}(q)$ between $\psi_{\lambda}(q)$ and $B_{\left[\Pi_{i} \alpha_{i}\right]}(q)$. Among the Bloch waves $B_{\left[\Pi_{i} \alpha_{i}\right]}(q)$, we note those bearing a single onsite excitation of order $\alpha_{j}>0$, all the other lattice sites $l$, being such as $\alpha_{l}=0$. For those states the label $\left[\Pi_{i} \alpha_{i}\right]$ reduces to $\alpha$. In case of $V_{\lambda, \alpha}(q)>0.5$, we choose to distinguish the eigenstate $\psi_{\lambda}$ by setting $\lambda=\alpha$. It simply means that the Bloch wave $B_{\alpha}(q)$ has a dominant contribution into $\psi_{\lambda}(q)$. At $C=0$, one notes that $V_{\alpha, \alpha}(q)=1$. As it may be expected, the scalar product $V_{\alpha, \alpha}(q)$ decreases as $C$ increases but its variation is smooth as found in Fig. 1 
FIG. 3: Energy spectrum of a $1 \mathrm{D}$ chain which model parameters are $A_{4}=0.2$ and $C=0.3$. The chain is composed of either $N=13$ sites. Two energy regions have been reported: (a) phonons and (b) quadriphonons. The eigen-energies are plotted as empty symbols and the phonon bound states energies have been signalized by full symbols.

(the results shown in this figure are examined thoroughly in the following). The eigenstates $\psi_{\alpha}(q)$ correspond to the $\alpha$ phonon bound states. That terminology may be rightly thought as ambiguous since a binding energy usually refers to a groundstate rather than to some excited states. However it is convenient as the excitation order $\alpha$ appears in the name. This order corresponds, indeed, to the energy level of the anharmonic on-site potential.

\section{PHONON BOUND STATES}

In lattices, treated in Ref. ${ }^{43}$, the sites number was $N=33$ for a basis cutoff $N_{c u t}=4$, which proves sufficient for the study of the two-phonon energy region. Here, we would like to extend our study to the case of a four phonon bound state (quadriphonon). We thus increased $N_{c u t}$ but the number of Bloch waves, involved in our basis for $N=33$, would overload our computer's memory, so we had to work with smaller lattices. For $N=13$ and $N_{\text {cut }}=6$, the rank of our basis reaches 6564 which can be managed within a reasonable time. We worked also with a even smaller lattice, $N=7$ which allows us to increase again $N_{\text {cut }}$ as large as $N_{\text {cut }}=9$. That case serves us as a reference in order to test the precision of our computations on the larger lattice.

Varying $C$ from the anti-continuous limit ${ }^{4}$, i.e., $C=0$ we plotted in Fig. 1 the eigenspectrum as a function of $C$. Every circle symbol represents a single eigenvalue in the half first Brillouin zone. The eigenvalues that correspond to the eigenstates $\psi_{\alpha}(q)$ (described in Sec have been plotted as full circles in Fig. 1, instead of empty ones for the other eigenstates. As far as we increased the coupling $C$ (see comment in Ref. ${ }^{47}$ ), in Fig 1, for a given order $\alpha$ and a fixed wave vector $q$, we found a unique eigenstate that verifies $V_{\lambda, \alpha}(q)>0.5$. This is the numerical proof that the nonlinear excitations may be continued from $C=0$ to larger coupling. This involves that the solutions $\psi_{\alpha}(q)$ conserve some features similar to the Bloch waves $B_{\alpha}(q)$. Such a behavior could have been expected ${ }^{43}$ while the energy gaps of the zero 
coupling spectrum remain. The hybridization between bound and unbound phonon states is, indeed, thought to be weak in that case. The point that was very unexpected is that even though $C$ is large enough for gaps to close (between the triphonon and the surrounding unbound phonon bands, for instance) we found a dominant contribution of $B_{\alpha}(q)$ into $\psi_{\alpha}(q)$. Moreover, for parameters in Fig 1 this property does not depend on the order of the excitation $\alpha$. It holds for phonons as for higher order phonon bound states. What differs, however, for the latter is their band width which increases with $C$ much smoother. In Figs 2 (a-d), at a fixed coupling, the eigenspectrum is plotted for different energy regions versus the wave vector. The same symbols as in Fig 1 are used. We note that the larger the energy is, the narrower the band of the phonon bound states is. Indeed, the phonon band width is about 0.06, whereas for the biphonon it is less than 0.01 , for triphonon it is around $10^{-3}$ and quadriphonon the band width falls to $10^{-5}$, in our energy unit. Although we approach only the very first energy excitations, up to the fourth order, we may reasonably extrapolate our results to higher energies. We then expect that the band width of the phonon bound states becomes exponentially narrower as the eigen-energy increases. In Figs 3 (a-b), the coupling parameter is such as the energy gaps close at high energy. We note that even though the energy spectrum exhibits no gap, we find some eigenstates $\psi_{\lambda}(q)$ that verify $V_{\lambda, \alpha}>0.5$ for $\alpha=4$. In that case, the binding energy of the so called $\alpha$ phonon bound states vanishes. However a strong component of $B_{\alpha}(q)$ takes part in $\psi_{\alpha}(q)$. For that reason, we propose to dub the $\psi_{\alpha}(q)$ eigenstates as nonlinear $\alpha$ phonons to emphasize that these states differ from the linear superposition of phonons, as well as to stress their quantized feature. In Fig 3 (b), the band width of the nonlinear four phonons is around 0.03 instead of $10^{-5}$ in Fig 2 (d). Although the width of that band increases substantially with the coupling, it is yet one order of magnitude below the phonon band width which is roughly 0.45 . The exponential decrease noted at low coupling seems to be no longer valid at larger coupling. This point deserves a thorough study that we propose to report in a future work. In Figs 2 (d) and 3 (b), the band of the quadriphonon does not exhibit the anomaly which appears when $N_{c u t}$ is diminished and that consists in a breaking of the band continuity. As far as $C<0.3$, our numerical approach seems to be reliable to treat the first phonon bound states. When the nonlinear parameter $A_{4}$ is small, i.e., of the order of $10^{-2}$ in our dimensionless model, the scalar product $V_{\lambda, \alpha}$ falls below $1 / 2$ for a sufficiently large $C$ which depends on $\alpha$. The larger $\alpha$ is, the larger the transition coupling $C_{\alpha}$ is. Moreover the $C_{\alpha}$ is found to depend on the 
wave vector $q$. At the edge of the lattice Brillouin zone, $C_{\alpha}$ is larger than in the center. In the limit where $A_{4}$ equals zero, the strictly harmonic eigenstates verify $V_{\lambda, \alpha}=0$ as soon as $C$ is switched on. In that particular case, $C_{\alpha}=0$ for all $\alpha$ but for non zero $A_{4}$, the $C_{\alpha}$ are larger than zero, even for $\alpha=1$ which corresponds to the single phonon.

In Ref. $\stackrel{46}{ }$, the author attempted to compare his theoretical computations, similar to Fig 1 . to some experimental measures in $\mathrm{H}_{2}{ }^{20}$ solid and $\mathrm{Ru}(100)$ : $\mathrm{CO}^{40}$. Although such a exercise was based on qualitative considerations, it is worthy to complete these comparison by noting that some spectral bands are due to the linear superposition of a biphonon and a single phonon (see Fig 1 in the present paper and Fig. 3 in Ref. ${ }^{46}$ ). The signature of these states has been measured in the IR spectrum of $\mathrm{HCl}$ solid 28 . Following a theoretical approach proposed earlier ${ }^{33}$, C. Gellini et al. carried out the computation ${ }^{28}$ of renormalized Green functions to interpret the $\mathrm{HCl}$ spectrum. Although such a theory would be inadequate for strong intersite coupling, for molecular crystals whose the molecule's bond anharmonicity dominates the inter-molecular coupling, as crystalline $\mathrm{CO}_{2}$ or $\mathrm{HCl}$ for instance, the renormalized Green functions seems relevant to capture the main physical properties. A convincing demonstration has been given by Bogani in Ref. ${ }^{48}$ where a precise fit of the IR adsorption spectrum has been achieved in several molecular crystals. The earlier work of V.M. Agranovich introduced initially the concept of phonon bound states and more specifically of biphonon for interpreting some experiments where anharmonic modes had been measured (see Ref. ${ }^{29}$ for infrared and Ref. ${ }^{49}$ for neutron spectra). The lattice model of Agranovich involves several energy terms that can be described briefly as follows. The elementary excitation is proportional to the on-site product of Bose-Einstein operators $a_{i}^{+} a_{i}$, while the tunneling between neighboring sites $i$ and $j$ is modelled by a hopping term $C a_{j}^{+} a_{i}$. The on-site Hubbard interaction between the boson pairs simulates the lattice anharmonicity by adding locally the energy operator $U\left(a_{i}^{+}\right)^{2} a_{i}^{2}$. Some other terms can be incorporated in the model to modify, for instance, the biphonon tunneling ${ }^{30}$ or the triphonon energy ${ }^{35}$. These energy contributions are parameterized by independent coefficients, e.g., $C, U$. For instance, the Hubbard model for boson has been used to interpret the INS in metal hydrides 35 . Here the model parameters have been adjusted to exhibit the same energy resonances as the INS spectrum. It is possible to achieve a similar work within the KG model, as shown in Ref. The Hubbard model for boson involves to neglect the energy terms that do not conserve the total boson number, despite the fact that these terms stem from the potential energy 
FIG. 4: Energy spectrum of a single anharmonic oscillator versus the eigenvalue rank for different parameters: (a) $A_{4}=0.2$ and (b) $A_{4}=0.4$. The semi-classical calculation (empty square symbols, dashed line) is compared to the Hamiltonian diagonalization (full circle symbols, solid line) onto the truncated Einstein basis (see Ref ${ }^{43}$ ) The $\mathrm{Y}$ axis unit is $\hbar \Omega$.

of atoms and molecules. The consequence of such an approximation is exemplified in computing the phonon dispersion law. For a one-dimensional lattice, the boson Hubbard lattice

would exhibit a phonon branch of the form $(1+C \cos (q))$, whereas the form $\sqrt{1+2 C \cos (q)}$ would be expected from the harmonic approximation with similar parameters. The latter case corresponds to the exact diagonalization of the linear KG Hamiltonian which includes only the quadratic potential energy of atoms. Since the two formula above diverge as $C$ increases, the Hubbard model for boson proves inappropriate to treat the normal modes at strong coupling. It might however be relevant to work out the high order nonlinear modes as proposed in Ref. 51 (see also the contribution of G.P. Tsironis in the present volume). Then the skipping of the non-conservative boson terms might find some substantiation in the fact that we found nonlinear narrow bands in continuous spectra as in Fig 1. A comparison between the boson Hubbard and our KG model would be very interesting to tentatively infer the properties of the high order phonon bound states.

\section{QUANTUM BREATHERS}

One introduces the time dependent Wannier state $W_{\alpha}(t, n)$, which is constructed from a combination of the $\alpha$ phonon bound states $\psi_{\alpha}(q)$. We recognize these eigenstates among others $\psi_{\lambda}(q)$ by the fact that they verify $V_{\lambda, \alpha}(q)>0.5$, for a fixed $\alpha$. This definition permits us to build a Wannier state even though the energy spectrum has no gap. Then the Wannier transform is written as follows:

$$
\left|W_{\alpha}(t, k)>=\frac{1}{\sqrt{N}} \sum_{q} e^{-i\left(q \times k+E_{\alpha}(q) \Omega t\right)}\right| \psi_{\alpha}(q)>.
$$

The subscript $k$ indicates the lattice site where is centered the Wannier transform. In Fig 1 , we found that the band of the $\alpha$ phonon bound states contains a single state per wave vector so that the sum over $q$ in the Wannier transform is complete. In case of a small intersite coupling, the Bloch wave $B_{\alpha}(q)$ is a good approximate of the $\alpha$ phonon bound state with 
the same wave vector. This may be thought as a Hartree approximation. To a first order in $C$, we found ${ }^{43}$ that the $E_{\alpha}(q)$ dependence on $q$ is negligible provided that $\alpha>1$ and $V$ is a single well potential. Then the Wannier state $\mid W_{\alpha}(t, k)>$ can be rewritten as:

$$
\mid W_{\alpha}(t, k)>=e^{-i\left(E_{\alpha} \Omega t\right)} \phi_{\alpha, k} \Pi_{l \neq k} \phi_{0, i} .
$$

Such a state is localized and time periodic so it may be considered as the quantum counterpart of the breather solutions for the classical nonlinear discrete KG lattice. These classical breather solutions have two important features that are first their spatial localization and second their time periodicity with a frequency and its overtones that are out of the linear classical phonon branch ${ }^{2}$. Our proposition could be verified by comparing the energies of a localized time periodic Wannier state and the semi-classical quantization of the classical breather orbits, in same lattice. In the simple case of zero inter-site coupling, such a comparison has been carried out in Ref. $\underline{\underline{43}}$ and Fig岛, for different on-site potentials. The remarkable agreement allows to expect that our proposition on breather quantum counterpart holds for larger values of $C$. To enforce our arguments, we dwell upon Fig 1, To a fixed $\alpha>1$, the energy of the corresponding Wannier state, given by the bracket of $H$, equals the mean energy $\tilde{E}_{\alpha}$, defined as the sum of $E_{\alpha}(q) / N$ over the first Brillouin zone. According to Fig 1, that mean energy does not vary much with $C$. Indeed, provided that $\alpha>1$ and $C<0.3$ (the upper boundary on $C$ to obtain a satisfactory precision), the bisecting line of the phonon bound states band is roughly parallel to the $\mathrm{X}$ axis. Consequently, the energy of the Wannier state of order $\alpha$ is comparable to the same quantity computed at $C=0$. In turn, the latter approaches very well the semi-classical quantization (see Fig (4) so the energy of the Wannier state and the one of semi-classical breather orbits do not differ in a significant manner provided that $C$ remains weak. It seems reasonable in the following to call $\alpha$ breather what is indeed the Wannier state of order $\alpha$. It would be worth carrying out the semi-classical quantization at non zero coupling in order to evaluate to what extend our expectations might be confirm. We think it should not contradict our arguments unless the classical breather becomes unstable, i.e., its frequency or one of its overtones fall in the spectrum of the classical normal modes.

We now study the dynamics of a $\alpha$ breather as a function of the order $\alpha$ and for a non zero coupling parameter. To that purpose, we integrate the time evolution of the on-site 
FIG. 5: The time evolution of kinetic energy of 17 atoms in one-dimensional KG chain, for a Wannier state made of phonon. The model parameters are $A_{4}=0.2, A_{3}=0$ and $C=0.05$. The lattice sites are reported on the $\mathrm{Y}$ axis while the $\mathrm{X}$ axis bears the time scale. The time unit is the inverse of $\Omega$.

(c)

FIG. 6: Profile of the 3D-plot described in Fig 5 for different breathers made of: (a) phonon, (b) biphonon, (c) triphonon and (d) quadriphonon. The parameters are same as in Fig 5 . The time is reported on the $\mathrm{X}$ axis. The $\mathrm{Y}$ axis unit is $\hbar \Omega$ ).

kinetic energy $P_{j}^{2} / 2$. The expectation of this operator is given by the bracket:

$$
<W_{\alpha}(t, k)\left|\frac{P_{j}^{2}}{2}\right| W_{\alpha}(t, k)>=\frac{1}{N} \sum_{q, q^{\prime}}<\psi_{\alpha}\left(q^{\prime}\right)\left|\frac{P_{j}^{2}}{2}\right| \psi_{\alpha}(q)>\times e^{-i\left(\left(q-q^{\prime}\right) \times k+\left(E_{\alpha}(q)-E_{\alpha}\left(q^{\prime}\right)\right) \Omega t\right)} .
$$

For $\alpha=1$, the Wannier state Eq.(5) is constructed from phonons. In the 3D plot (see Fig (5) of the kinetic energy time evolution, one notes that the energy is initially localized and quickly spreads over the lattice. In Fig 6 (a), the profile of the 3D plot shows that after 40 time unit, the energy is no longer localized. The same profile plot for $\alpha=2$, in Fig[6] (b) shows that the quantum breather made of biphonons may last 10 times longer than for phonons. With the Wannier transform of triphonons, the life time of the localized excitation is again raised by one order of magnitude (see Fig [6 (c)). The life time of the fourth quantum breather (i.e., the Wannier transform of the quadriphonons) overpasses the first case in Fig [6] (a), by three orders. Conclusively, we found that the nonlinear KG lattice may sustain a high energy spike for longer than $10^{3}$ times the typical relaxation of low energy excitations, imposed by phonons. This behavior is related to the dispersion of the phonon bound states since the thinner the band is, the longer the Wannier transform remains coherent. According to our results, the breather life time increases exponentially with respect to $\alpha$. As noted previously (see Sec III), the band width decreases as the order of phonon bound states increases, even though at high energy the spectrum becomes continuous (see Fig (1). According to our results, this continuity does not involve a particular decay in the breather life time. It is noteworthy that the decay of a quantum breather is athermal as it stems from the decoherence of the phonon bound states. 
FIG. 7: The same as in Fig 6 but for $C=0.3$ and different sizes: (a-b) $N=13$ and (c-d-e) $N=7$. The Wannier states are made of either (a-c) phonon, (b-d) quadriphonon or (e) pentaphonon. The time is reported on the $\mathrm{X}$ axis and the $\mathrm{Y}$ axis unit is $\hbar \Omega$. In the insets, some distinct time intervals have been magnified.

As $C$ increases, the $\alpha$ breather life time decreases, in agreement to the band width enlargement shown in Fig,1. This can be worked out from the comparison of Fig. 6 (a) and Fig. 6] (d) for $C=0.05$ to the left hand side insets in Fig. [7 (a) and Fig. [7 (b) for $C=0.3$. Each couple of figures concern the cases $\alpha=1$ and $\alpha=4$. For $\alpha=1$, one sees that the life time of the localized excitation decreases from 40 to 4 time units while for $\alpha=4$, the breather life time decreases from $8 \times 10^{4}$ to $8 \times 10^{1}$. The drop is sharper for the higher order. However for a fixed value of $C$, whatever this value is, the band width of phonon bound states decreases as the energy increases so that the life time of the corresponding breather increases too. In Fig. 7 (e), the life time of the fifth breather is still two order of magnitude larger than for $\alpha=1$. We thus expect that for a sufficiently high energy spike, the breathing mode survives noticeably even though the intersite coupling is large.

For a fixed order $\alpha$, the breather life time does not depend on the lattice size, as found in comparing Fig. 7 (a) to Fig. 7 (c) and Fig. 7 (b) to Fig. 7(d). The Figs. 7 (a-b) have been obtained for a 13 sites lattice and the Figs. 7 (c-d) for a 7 sites lattice. Another interesting feature revealed by these results is the time recurrence of breather. Indeed, one notes that a certain time after the energy spike has spread, the energy backs to its initial trapped state, similar to the original one (see right hand side insets in Figs. 7 (a-e)). The breather is then bear by few sites although it has not exactly the same amplitude as initially. The retrapping process occurs for a time twice larger than the breather life time because of the time inversion symmetry. According to our computations, there is no exact frequency for the breather recurrence as no regular behavior may be depicted in Figs. 17(a-e). Though, we note that the recurrence occurs sooner in a smaller lattice, which is demonstrated by comparison either of Fig. 7 (a) to (c) or Fig. 7] (b) to (d). In a macroscopic crystal, the recurrence is thus expected never to take place. In contrast, the breather recurrence might occur in a single molecule as benzen. To that respect, the breather recurrence might be worth studying thoroughly. Eventually, the shortest time interval upon which the recurrence occurs after 
starting the breather dynamics seems to increase with $\alpha$ as shown by comparing Fig. [7 (c) to Fig. 7 (d) or Fig. 7 (d) to Fig. 7 (e).

\section{CONCLUSION AND POSSIBLE DEVELOPMENTS}

As a summary, we attempted to work out the breather modes in the quantum KG lattice. We provided a numerical method to estimate their life time and spatial expansion. At the quantum scale, it proves that the breathers are closely related to what has been called earlier, the phonon bound states that are anharmonic eigenmodes. It is, indeed, well-known in condensed matter physics that a narrow band excitations may be viewed as a quasi-particle through a Wannier transform. We applied that theory to the phonon bound states and showed that the lattice may sustain the corresponding breather for a time which increases as the magnitude of the energy spike. At low intersite coupling, we found that the breather life time increases exponentially with the trapped energy. This variation softens at larger coupling, mainly because of the hybridization between the phonon bound states and the linear superpositions of lower energy modes. For seak of simplicity, we only treated a quartic nonlinearity. We found nonlinear excitations for all couplings we tested, i.e., up to $C=0.3$ which corresponds to a dispersion that is larger than the anharmonicity. In the classical counterpart of our KG lattice, a similar result is obtained since the discrete breathers occur at all coupling too because their frequency is higher than the normal modes band. However, the cubic nonlinearity is known to modify significantly this feature as the breather frequency should be smaller than the normal modes. Consequently, for a given breather solution, that is for a fixed frequency, there is a coupling threshold above which the breather is no longer stable. This transition occurs when the breather frequency or one of its overtones fall into the classical normal band ${ }^{2}$. A similar behavior is expected in the quantum case, which will be studied in a future work.

The results we obtained in a KG lattice are rather encouraging for a possible future study of the quantum acoustic lattices, as the FPU 52 chain. Even though we did not address precisely that case, we found that in energy spectra where no gap occurs, the nonlinear excitations may yet be distinguished and still exhibit a particle-like energy branch. Such excitations are expected to emerge in the quantum FPU chain too, under the condition that they corresponds to a sufficiently large energy. Our numerical theory should be tractable 
on the one-dimensional FPU lattice with only few sites, even though the rank of our basis might increase dramatically. Then, one could yet achieve the Hamiltonian diagonalization with a iterative procedure as the Lanczos method.

Alongside the present work, we carried out the calculation of the dynamical structure factor of the nonlinear $\mathrm{KG}$ lattice ${ }^{50}$. A simulation of the inelastic scattering has been achieved so as to compare our theory to practical cases.

\section{Acknowledgments}

I gratefully acknowledge S. Aubry who introduced me to the theory of breathers, at coffee breaks in Laboratoire Léon Brillouin (CEA-Saclay) .

* Electronic address: lproville@cea.fr

1 A. J. Sievers and S. Takeno, Phys. Rev. Lett. 61, 970 (1988); S. Takeno, K. Kisoda and A. J. Sievers, Prog. of Theor. Phys. Suppl. 94, 242 (1988).

2 R.S. MacKay and S. Aubry, Nonlinearity 7, 1623 (1994).

3 W.Z. Wang, J.T. Gammel, A.R. Bishop and M.I. Salkola, Phys. Rev. Lett. 76, 3598 (1996).

4 S. Aubry, Physica D 103, 201 (1997).

5 T. Cretegny, T. Dauxois, S. Ruffo and A. Torcini, Physica D 121, 109 (1999).

6 R.S. Mackay, Physica A 288174 (2000).

7 G. Kopidakis and S. Aubry, Phys. Rev. Lett. 84, 3236 (2000).

8 V. Hizhnyakov, D. Nevedrov and A. J. Sievers, Physica B 316-317, 132 (2002).

9 J. Dorignac and S. Flach,Phys. Rev. B 65, 214305 (2002); V. Fleurov,R. Schilling and S. Flach, Phys. Rev. E 58, 339 (1998).

10 J. C. Eilbeck, Proceedings of the 3rd Conference Localization and Energy Transfer in Nonlinear Systems, Edited by L. Vazquez, R. S. MacKay and M. P. Zorzano (World Scientific, Singapore, 2003), p. 177.

11 P. Rosenau and S. Schochet, Phys. Rev. Lett. 94, 045503 (2005). 
12 S. Tretiak, A. Piryatinski, A. Saxena, R. L. Martin, and A. R. Bishop Phys. Rev. B 70, 233203 (2004).

13 J. Gómez-Gardeñes, L. M. Flora, M. Peyrard, and A. R. Bishop Chaos 14, 1130 (2004).

14 V. Fleurov, Chaos 13, 676 (2003).

15 J.J. Mazo and T.P. Orlando, Chaos 13, 733 (2003).

16 A. C. Scott and J. C. Eilbeck, Chemical Physics Letters 132, 23 (1986).

17 S. Adachi, V.M. Kobryanskii and T. Kobayashi, Phys. Rev. Lett. 89, 027401 (2002).

18 S.P. Love, L.A. Worl, R.J. Donohoe, S.C. Huckett and B.I. Swanson, Phys. Rev. B 46, 813 (1992);

B.I. Swanson, J.A. Brozik, S.P. Love, G.F. Strouse, A.P. Shreve, A.R. Bishop, W-Z. Wang and M.I. Salkola, Phys. Rev. Lett. 82, 3288 (1999);

N.K. Voulgarakis, G. Kalosakas, A.R. Bishop and G.P. Tsironis, Phys. Rev. B 64, 020301(R) (2001).

19 M.P. Gush, W.F. Hare, E.J. Allin and H.C. Welsh, Phys. Rev. 1061101 (1957).

20 H. Mao and R.J. Hemley, Rev. Mod. Phys. 66, 671 (1994).

21 J. V. Kranendonk and G. Karl, Rev. Mod. Phys. 40, 531 (1968).

22 N.B. Abbott and A. Elliot, Proc. R. Soc. London A 234, 247 (1956).

23 J. Edler and P. Hamm, J. Chem. Phys. 119, 2709 (2003).

24 J. Edler and P. Hamm, Phys. Rev. B 69, 214301 (2004).

25 J. Edler and P. Hamm, Phys. Rev. Lett. 93, 106405 (2004).

26 A. C. Scott, Nonlinear science, (Oxford, New York, 2003) , Chap. 8, pp. 337-420.

27 A. Ron and D.F. Hornig, J. Chem. Phys. 39, 1129 (1963).

28 C. Gellini, P.R. Salvi and V. Schettino, J. Chem. Phys. 106, 6942 (1997).

29 V.M. Agranovich, Soviet Physics - Solid State 12, 430 (1970).

30 V.M. Agranovich, Spectroscopy and Excitation Dynamics of Condensed Molecular Systems, (North-Holland, New York, 1983), Chap. 3, pp. 83-138.

31 D.A. Dows and V. Schettino, J. Chem. Phys. 58, 5009 (1975);

V. Schettino and P.R. Salvi, Spectrochim. Acta 31A, 399 (1975).

32 D. Kroh and A. Ron, Chem. Phys. Lett. 36, 527 (1975).

33 F. Bogani, J. Phys. C 11, 1283 (1978); J. Phys. C 11, 1297 (1978).

34 D. K. Ross, V. E. Antonov, E. L. Bokhenkov, A. I. Kolesnikov, and E. G. Ponyatovsky, Phys. 
Rev. B 58, 2591 (1998).

A.I. Kolesnikov, M. Prager, J. Tomkinson, I.O. Bashkin, V. Yu Malyshev and E.G. Ponyatovskii, J. Phys.: Condens. Matter 3, 5927 (1991);

A.I. Kolesnikov, A.M. Balagurov, I.O. Bashkin, A.V. Belushkin, E.G. Ponyatovsky and M. Prager, J. Phys.: Condens. Matter 6, 8977 (1994);

A.I. Kolesnikov, I.O. Bashkin, A.V. Belushkin, E.G. Ponyatovskii, and M. Prager, J. Phys.: Condens. Matter 6, 8989 (1994);

I.O. Bashfkin, A.I. Kolesnikov and M.A. Adams, J. Phys.: Condens. Matter 12, 4757 (2000).

36 G.J. Kearley, F. Fillaux, M.-H. Baron, S. Bennington and J. Tomkinson, Science 264, 1285 (1994).

37 F. Fillaux, B. Nicolaia, W. Paulus, E. Kaiser-Morris and A. Cousson, Phys. Rev. B 68, 224301 (2003).

38 F. Fillaux and C.J. Cardile, Phys. Rev. B 425990 (1990).

39 J.A.D. Wattis, Physica D 82, 333 (1995).

40 P. Jakob and B.N.J. Persson, J. Chem. Phys. 109, 8641 (1998).

41 M. Bonn, C. Hess and M. Wolf, J. Chem. Phys. 115, 7725 (2001).

42 V. Pouthier, J. Chem. Phys. 118, 9364 (2003).

43 L. Proville, Phys. Rev. B 71, 104306 (2005).

44 C. Elsasser, K.M. Ho, C.T. Chan and M. Fahnle, J. Phys.: Condens. Matter 4, 5207 (1992).

45 William H. Press, Numerical Recipes in Fortran, Cambridge University Press (1992), pp. 462$475 ;$ http://www.nr.com/.

46 L. Proville, EuroPhys. Lett. 69, 763 (2005).

$47 C \approx 0.5$ corresponds to a acoustic lattice.

48 F. Bogani, J. Phys. C: Solid State Phys. 11, 1297 (1978).

49 V.M. Agranovich and I.I. Lalov, Soviet Physics - Solid State 18, 1148 (1976).

50 L. Proville, to be published (2005).

51 O.A. Dubovskiï and A.V. Orlov, Physics of Solid State 36, 3131 (1994).

52 N. J. Zabusky, Chaos 15, 015102 (2005) 
This figure "Figure1.png" is available in "png" format from: http://arxiv.org/ps/quant-ph/0507033v1 
This figure "Figure2a.png" is available in "png" format from: http://arxiv.org/ps/quant-ph/0507033v1 
This figure "Figure2b.png" is available in "png" format from: http://arxiv.org/ps/quant-ph/0507033v1 
This figure "Figure2c.png" is available in "png" format from: http://arxiv.org/ps/quant-ph/0507033v1 
This figure "Figure2d.png" is available in "png" format from: http://arxiv.org/ps/quant-ph/0507033v1 
This figure "Figure3a.png" is available in "png" format from: http://arxiv.org/ps/quant-ph/0507033v1 
This figure "Figure3b.png" is available in "png" format from: http://arxiv.org/ps/quant-ph/0507033v1 
This figure "Figure4.png" is available in "png" format from: http://arxiv.org/ps/quant-ph/0507033v1 
This figure "Figure5.png" is available in "png" format from: http://arxiv.org/ps/quant-ph/0507033v1 
This figure "Figure5a.png" is available in "png" format from: http://arxiv.org/ps/quant-ph/0507033v1 
This figure "Figure5b.png" is available in "png" format from: http://arxiv.org/ps/quant-ph/0507033v1 
This figure "Figure5c.png" is available in "png" format from: http://arxiv.org/ps/quant-ph/0507033v1 
This figure "Figure5d.png" is available in "png" format from: http://arxiv.org/ps/quant-ph/0507033v1 
This figure "Figure6a.png" is available in "png" format from: http://arxiv.org/ps/quant-ph/0507033v1 
This figure "Figure6b.png" is available in "png" format from: http://arxiv.org/ps/quant-ph/0507033v1 
This figure "Figure6c.png" is available in "png" format from: http://arxiv.org/ps/quant-ph/0507033v1 
This figure "Figure6d.png" is available in "png" format from: http://arxiv.org/ps/quant-ph/0507033v1 
This figure "Figure6e.png" is available in "png" format from: http://arxiv.org/ps/quant-ph/0507033v1 
This figure "Figure55.png" is available in "png" format from: http://arxiv.org/ps/quant-ph/0507033v1 